\title{
Completion of the Genome Sequence of Watermelon silver mottle virus and Utilization of Degenerate Primers for Detecting Tospoviruses in Five Serogroups
}

\author{
Fang-Hua Chu, Chia-Hung Chao, Min-Hsun Chung, Ching-Chung Chen, and Shyi-Dong Yeh
}

First, third, and fifth authors: Department of Plant Pathology, National Chung Hsing University, Taichung, Taiwan, R.O.C.; and second and fourth authors: Taichung District Agricultural Improvement Station, Changhua, Taiwan, R.O.C. Accepted for publication 4 January 2001.

\begin{abstract}
Chu, F.-H., Chao, C.-H., Chung, M.-H., Chen, C.-C., and Yeh, S.-D. 2001. Completion of the genome sequence of Watermelon silver mottle virus and utilization of degenerate primers for detecting tospoviruses in five serogroups. Phytopathology 91:361-368.

The nucleotide sequence of the L RNA of Watermelon silver mottle virus (WSMoV) was determined. Combined with the previous work on $\mathrm{M}$ and S RNAs, the whole genomic sequence of this member of the genus Tospovirus was completed. The L RNA is 8,917 nucleotides in length, with one large open reading frame encoding a translation product of 2,878 amino acids $(331.8 \mathrm{kDa}$ ) on the viral complementary strand. The $\mathrm{L}$ protein shares amino acid identities of only 44.3 and $46.5 \%$ with Tomato spotted wilt virus (TSWV) and Impatiens necrotic spot virus, respectively; but an amino acid identity of $91.3 \%$ with Peanut bud necrosis virus. Among the sequenced tospoviruses, $\mathrm{L}$ protein was the most conserved gene product, whereas the nonstructural S protein was generally

the most variable. Comparison of the deduced $\mathrm{L}$ protein of WSMoV with those of other members of the family Bunyaviridae revealed that its amino acid sequence includes the reported conserved motifs of RNAdependent RNA polymerases. To develop a method for detecting tospoviruses by reverse transcription-polymerase chain reaction (RT-PCR), two pairs of degenerate primers were designed from conserved regions of the $\mathrm{L}$ genes and used to amplify the corresponding regions of the $\mathrm{L}$ genes from total RNAs extracted from plant tissues infected with five serologically distinct tospoviruses. The DNA fragments obtained were identified as those of tospoviruses by restriction enzyme digestion and DNA sequencing. For field samples, watermelon and wax gourd infected with WSMoV, and lisianthus infected with TSWV were also successfully detected by these two pairs of degenerate primers, with a sensitivity similar to N-gene-specific primers. The results indicated that the RT-PCR with the degenerate primers is a fast and reliable method for detecting tospoviruses in different serogroups.
\end{abstract}

The family Bunyaviridae includes five genera, Bunyavirus, Phlebovirus, Hantavirus, Nairovirus, and Tospovirus (12,28). Tospovirus is the only genus of the family that infects plants. At least one tospovirus is transmitted in nature by thrips in a propogative and persistent manner (43). These viruses have quasispherical-enveloped particles containing three single-stranded RNA (ssRNA) segments denoted S, M, and L RNAs $(26,44)$. The L RNA is of negative polarity and encodes a putative RNA polymerase that may be associated with the ribonucleoprotein complex $(3,9,18,45)$. The other two genomic RNAs use an ambisense coding strategy. The M RNA encodes a nonstructural $(\mathrm{NSm})$ protein in the viral $(\mathrm{v})$ sense and a protein to serve as the precursor for the G1 and G2 glycoproteins in the viral complementary (vc) sense $(7,17,23,35)$. The NSm protein may be involved in cell-to-cell movement of nonenveloped ribonucleocapsid structures (20). Tubular structures are specifically formed when the NSm gene is expressed in both plant and insect cell systems (39). The G1 and G2 glycoproteins are believed to form spikes on the viral envelope $(26,40)$. The $\mathrm{S}$ RNA encodes in the $\mathrm{v}$ sense a nonstructural (NSs) protein that forms filamentous inclusion bodies (19) and in the vc sense a nucleocapsid protein (NP) that encapsidates viral RNAs $(8,22)$.

Watermelon silver mottle virus (WSMoV) is the most important pathogen in crops of watermelon and other cucurbits in Taiwan

Corresponding author: S.-D. Yeh; E-mail address: sdyeh@dragon.nchu.edu.tw

The nucleotide sequence reported used GenBank Accession No. AF133128.

Publication no. P-2001-0213-01R

(C) 2001 The American Phytopathological Society
$(47,49)$. Both WSMoV and Peanut bud necrosis virus (PBNV) are transmitted by Thrips palmi Karny $(31,49)$, and are serologically related to another tospovirus, Watermelon bud necrosis virus (WBNV), which is transmitted by Thrips flavus Schrank (37). These three tospoviruses cause serious damage on peanut (PBNV) and cucurbits (WSMoV and WBNV) in Asia and are classified as serogroup IV tospoviruses $(2,37,49)$. The NP of WSMoV is serologically unrelated to those of serogroup I (Tomato spotted wilt virus, TSWV), serogroup II (Groundnut ringspot virus, GRSV; Tomato chlorotic spot virus, TCSV), and serogroup III (Impatiens necrotic spot virus, INSV) $(2,47)$.

The complete genome sequence has been reported for three tospovirus species, including TSWV of serogroup I $(9,10,17)$, INSV of serogroup III $(8,23,45)$, and PBNV of serogroup IV $(16,34,35)$. Both $\mathrm{S}$ and $\mathrm{M}$ segments of the tripartite RNA genome of WSMoV have been previously sequenced by our laboratory $(7,50)$, and in this paper we report the nucleotide sequence of the full length WSMoV L RNA. We also report the phylogenetic relationships of the $\mathrm{L}$ proteins based on interserogroup and intraserogroup in the genus Tospovirus and the phylogenetic relationships of the $\mathrm{L}$ protein of WSMoV with viruses in the other genera of the family Bunyaviridae. Furthermore, we developed a fast and reliable method for the detection of tospoviruses in five different serogroups by reverse transcription-polymerase chain reaction (RT-PCR) with degenerate primers designed from the conserved regions of $\mathrm{L}$ genes.

\section{MATERIALS AND METHODS}

Virus sources and isolation of $L$ double-stranded RNA. An isolate of WSMoV, collected from watermelon (Citrullus lanatus 
Thunb., Matsum. \& Nakai) in Taiwan, was used for the study (49). A typical isolate of TSWV (serogroup I) obtained from tomato in New York (denoted TSWV-NY, provided by R. Provvidenti, New York State Experiment Station, Geneva), a typical isolate of GRSV (serogroup II) collected from Brazil (denoted GRSV-B, provided by D. Gonsalves, New York State Experiment Station), and a typical isolate of INSV (serogroup III) collected from impatiens in the United States (denoted INSV-M, provided by J. Moyer, North Carolina University, Raleigh) were also used for comparison. A high temperature-recovered (HT-1) tospovirus isolate (serogroup IV) from gloxinia was obtained from $\mathrm{H}$. T. Hsu (U.S. Department of Agriculture, Beltsville, MD). An isolate of Peanut chlorotic fan-spot virus (PCFV, an independent serogroup distinct from serogroup I, II, III, and IV) (48) collected from peanut was provided by C. C. Chen (Taichung District Agricultural Improvement Station, Datsuen, Taiwan). These viruses were transmitted to, and maintained in, the systemic host Nicotiana benthamiana Domin., and the local lesion host Chenopodium quinoa Willd., by mechanical inoculation.

The procedure for isolation of double-stranded RNA (dsRNA) of WSMoV from virus-infected plants of Chenopodium quinoa was similar to that described by Yeh and Chang (47). The L dsRNA of WSMoV was separated by electrophoresis, eluted from the agarose gel, and used for the construction of a cDNA library.

Construction of the cDNA library to L dsRNA and hybridization selection. The eluted L dsRNA of WSMoV was denatured by methylmercuric hydroxide (MeHg) (5). A cDNA library was constructed according to the protocols for ZAP-cDNA synthesis and cloning (Stratagene, La Jolla, CA). The dsDNA was inserted into lambda ZAP-II EcoRI/calf intestinal alkaline phosphatase vector (Stratagene) and packaged with capsid proteins (Gold Packaging Extract; Stratagene).

The cDNA clones to the L RNA were screened by plaque hybridization according to the method of Maniatis et al. (24). The ${ }^{32} \mathrm{P}$-labeled cDNA probe used for screening was synthesized from $\mathrm{MeHg}$-denatured L dsRNA by random priming according to Maniatis et al. (24). Additionally, ${ }^{32} \mathrm{P}$-labeled probes nick-translated (24) from the selected cDNA clones were used to further screen other clones from the library.

Cloning of the $5^{\prime}$ and $3^{\prime}$ terminal regions. Terminal regions of the L RNA were cloned by RT-PCR with the isolated L RNA of WSMoV. The first-strand cDNA was synthesized by SuperScript II RT (Gibco BRL, Gaithersburg, MD), and DNA amplified by PCR with ExTaq DNA polymerase (TaKaRa, Shiga, Japan). PCR products were cloned using the TA cloning kit (Invitrogen, Carlsbad, CA). Primers used for amplifying unknown regions were designed from the known sequences of WSMoV L RNA and the reported sequences of L RNAs of other tospoviruses: TSWV (D10066) (9), INSV (X93218) (45), and PBNV (AF025538) (16). The corresponding positions of the primers on the $\mathrm{v}$ strand of the L RNA are indicated in Figure 1. The 5'terminal fragment was amplified by a specific primer, wl4-7, designed from the known WSMoV L RNA sequence (5'-GGCCTCAAATGATTGCTATGAGC-3', nucleotide positions 2297 to 2319) and a primer, consL, designed from the terminal consensus sequence of the above tospoviruses (5'-AGAGCAATCGTGCAAC-3', position 1 to 16). For the $3^{\prime}$ end of the L RNA, the RT-PCR-amplified region reflecting positions 7769 to 8917 was obtained by degenerate primer b1 (5'-ATG(G/T/C)(A/T)G(A/G)TCA(C/T)TCAT(G/T)$\left.\mathrm{GT}(\mathrm{C} / \mathrm{T}) \mathrm{TC}-3^{\prime}\right)$ and the primer consL encoding the terminal consensus sequence. The region from position 5917 to 7589 was obtained with specific primer wl34-1c (5'-CTATAGTCTTCAATGTTGATCCTG-3'), designed from the known sequence, and degenerate primer b2 (5'-GGAAATAGAAAGCATTGAACG(T/A)(G/T/C)(A/C)-3'). The region from positions 7215 to 7873 that covered the locations of degenerate primers b1 and b2 was obtained by two specific primers (wb2-1c; 5'-CATTTCTTGTCTTCACGTCTCC-3' and wl5-1c; 5'-GATCTAATT-
GATGCTATGCC-3') designed from the known cDNA sequences of the L RNA.

To confirm the terminal sequences of the L RNA, the firststrand cDNAs were synthesized by the primer wl3-2 (5'-AGCTTGTGAAGCCTGCC-3', positions 1186 to 1202) for the $5^{\prime}$ end and the primer wb2-1c for the $3^{\prime}$ end. These were then tailed with dCTP and terminal deoxynucleotidyl transferase (Gibco BRL), and the terminal regions were amplified by PCR by an upstream primer (g12 of 5'-CGACTAGTGGGGGGGGGGGG-3') and a second downstream specific primer (wl3-4 of $5^{\prime}$-AGCTCATATCTGATACCTGC- $3^{\prime}$ positions 825 to 844 for the $5^{\prime}$ end, and the degenerate primer b1 for the $3^{\prime}$ end).

Computer analysis of the sequence. DNA sequencing was determined by the dideoxynucleotide chain termination method (33) using Sequenase (version 2.0, DNA Sequencing Kit; United States Biochemical Corp., Cleveland) or an automatic DNA sequencing system (ABI 377-19; Perkin-Elmer Applied Biosystems, Foster City, CA). Nucleotide sequences were assembled and the encoded amino acid sequences were predicted using PC/GENE 6.85 software (IntelliGenetics Inc., University of Geneva, Switzerland). Sequence comparisons were made with the GAP program of the Genetics Computer Group (GCG) package (version 9.0; Genetics Computer Group, Madison, WI). The PILEUP program from the GCG package was used to align sets of multiple sequences. Matrices of the dissimilarities of aligned protein sequences were calculated using the Jukes-Cantor correction. Phylogenetic relationships were calculated from these distances by the unweighted pair group method with arithmetic and represented as trees using the GROWTREE program. Sequences for comparisons were obtained from the EMBL Database under the following accession codes: TSWV, D10066 (9); INSV, X93218 (45); PBNV, AF025538 (16); Bunyamwera (BUN), X14384 (11); La Crosse (LAC), U12396 (32); Toscana (TOS), X68414 (1); Rift Valley fever (RVF), X56464 (27); Unkuniemi (UUK), D10759 (13); Puumala strain Sotkamo (PUUs), Z66548 (29); Puumala strain Bashkiria (PUUb), M63194 (38); Sin Nombre (SN), L37901 (6); Hantaan (HTN), X55901 (36); Seoul (SEO), X56492 (4); and Tula (TUL), AJ005637 (21).

Design of degenerate primers for virus detection from the conserved regions of $\mathbf{L}$ RNA. For identification of tospoviruses of different serogroups, degenerate primers were designed from the conserved regions of the $\mathrm{L}$ genes of tospoviruses. Two pairs of conserved regions of $\mathrm{L}$ genes of WSMoV and other published tospoviruses $(9,16,45)$ were identified for designing primers. Degenerate primer gL3637 with the sequence 5'-CCTTTAACAGT(A/T/G)GAAACAT-3' (positions 3637 to 3655 of the vc sense of WSMoV L RNA) was used as an upstream primer. Primer gL4435c with the sequence $5^{\prime}$-CAT(A/T/G)GC(A/G)CAAGA(A/G)TG(A/G)TA(A/G)ACAGA-3' (positions 4435 to 4456 of the vc sense of WSMoV L RNA) and gL4510c with the sequence $5^{\prime}$ TCATC(A/G)GA(A/G)TG(T/G/C)AC(A/C)ATCCATCT-3' (positions 4510 to 4532 of the vc sense of WSMoV L RNA), which were complementary to the nucleotide sequences encoding for the conserved amino acid, were used as two individual downstream primers.

RNA extraction and RT-PCR conditions. Total RNAs extracted from plants individually infected with a panel of viruses were subjected to RT-PCR using the designed degenerate primers. The panel included three tospoviruses, TSWV (9), INSV (45), and WSMoV, in which the sequences of the $\mathrm{L}$ genes have been determined, and three tospoviruses, GRSV, HT-1, and PCFV, in which the sequences of $\mathrm{L}$ genes have not been determined. As controls, Odontoglossum ringspot virus (ORSV) a member of the genus Tobamovirus, Potato virus $X$ (PVX) genus Potexvirus, Papaya ringspot virus (PRSV) genus Potyvirus, Strawberry latent ringspot virus (SLRV) genus Nepovirus, Cucumber mosaic virus (CMV) genus Cucumovirus, Rice grassy stunt virus (RGSV) genus Tenuivirus, Rice stripe virus (RSV) genus Tenuivirus, and 
healthy plants were included. Total RNAs were extracted from virus-infected plants as advised in the manufacturer's protocol for RNA isolation (ULTRASPEC; Biotecx Company, Houston) and used for RT-PCR. Test samples included plants of $N$. benthamiana infected with individual tospoviruses; plants of Chenopodium quinoa infected with ORSV, PVX, SLRV, or CMV; plants of Carica papaya L., infected with PRSV; and plants of Oryza sativa L., infected with RGSV or RSV. The plant tissues individually infected with ORSV, PVX, or SLRV were donated by C. A. Chang (Taiwan Agricultural Research Institute, Wufeng, Taiwan). The plant tissues infected with RGSV or RSV were donated by C. C. Chen (Taichung District Agricultural Improvement Station, Datsuen, Taiwan). Each test sample tissue was checked for a positive reaction by enzyme-linked immunosorbent assay (ELISA) with the corresponding antiserum and by mechanical or insect transmission to the corresponding test plants to ensure the presence of the viruses. RT was performed with 1 to $2 \mu \mathrm{g}$ of total RNA extracted from leaves and designed primers in a reaction volume of $50 \mu \mathrm{l}$ (Fast-Run RT-PCR; Proteck, Taipei, Taiwan). Mixtures were incubated at $50^{\circ} \mathrm{C}$ for $40 \mathrm{~min}$ to synthesize the first strand cDNA, and heated at $94^{\circ} \mathrm{C}$ for 2 min to denature RT. PCR was performed with 30 cycles of $30 \mathrm{~s}$ for strand separation at $94^{\circ} \mathrm{C}, 30 \mathrm{~s}$ for annealing at $50^{\circ} \mathrm{C}$, and $2 \mathrm{~min}$ for synthesis at $72^{\circ} \mathrm{C}$.

Confirmation by restriction and sequencing. PCR products were digested with HindIII, XbaI, and EcoRI, and the digested fragments were analyzed by electrophoresis in $2 \%$ agarose gel to check the accuracy of the amplification. To verify the sequences of the DNA products, PCR products were cloned (Invitrogen), and the nucleotide sequences of the amplified fragments were determined by the dideoxy chain termination method (33). The sequences of the PCR products were aligned by PILEUP and PRETTY (GCG) programs.

Detection of diseased samples from field. Diseased samples of watermelon (15 samples), wax gourd (Benincasa hispida Gogn.) (12 samples), and lisianthus (Eustoma grandiflorum Raf., Shinn) (3 samples) with tospovirus-like symptoms were collected from the field to evaluate the feasibility of using the two pairs of degenerate primers derived from L RNAs of the tospoviruses for detection of tospoviruses by RT-PCR. These samples were also analyzed by RT-PCR with specific primers to $\mathrm{N}$ genes and specific antisera to NPs by indirect ELISA. The two pairs of degenerate primers derived from L RNAs of the tospoviruses were previously described. The WSMoV-N-gene-specific primers were 5'-ACAGAAAGGTTAGCACTGAA-3' and 5'-ACAGAGGACTCCACTCCCGG-3', amplifying a DNA fragment of $0.7 \mathrm{kbp}$ (47). The TSWV-N-gene-specific primers were 5'-CGGGATCAGAGCAATTGTGTCA-3' and 5'-GCGAGCTCTTAACACACTAAGCAAGCAC-3', amplifying a DNA fragment of $1.0 \mathrm{kbp}$ (10). Extraction of total RNAs and the procedure for RT-PCR were the same as described for the greenhouse samples. Each antiserum to the purified NP of WSMoV or TSWV was previously produced in our laboratory, and the procedure for indirect ELISA was described previously (48).

\section{RESULTS}

Sequence determination and analysis of the L RNA. The positions of the selected cDNA clones representing the whole L RNA molecule are summarized in Figure 1. Five clones (L3, L4, L28, L34, and L44) were selected by hybridization with cDNA probes derived from the L dsRNA of WSMoV. The clones WL3, amplified by primers consL/w14-7, LB2, amplified by primers wl34-1c/b2, WL5, amplified by primers b1/consL, and LP1, amplified by primers wb2-1c/wl5-1c, were obtained by RT-PCR. The sequence determined from clone LP1 clarified the sequence ambiguities at the loci covered by degenerate primers b1 and b2. To confirm the terminal sequences of the L RNA, clone GL5, amplified by primers g12/wl3-4, and clone GL3, amplified by primers b1/g12, were sequenced.

The sequence assembled from the above clones represented the complete viral L RNA of WSMoV. It was 8,917 nucleotides (nts) in length and had a base composition of $29.0 \% \mathrm{~A}, 18.7 \% \mathrm{C}$, $15.4 \% \mathrm{G}$, and $36.9 \% \mathrm{U}$. The L RNA of WSMoV was 20,141 , and 6 nts longer than those of TSWV (8,897 nts), INSV (8,776 nts), and PBNV (8,911 nts), respectively. The L RNA of WSMoV had overall nucleotide identities of 55.1, 55.5, and $81.1 \%$ with those of TSWV, INSV, and PBNV, respectively.

Computer-predicted reading frames of the $\mathrm{v}$ and $\mathrm{vc}$ strands of the L RNA of WSMoV revealed that there was only one large open reading frame (ORF), which was located on the vc strand and similar to that found in the reported L RNAs of other tospoviruses $(9,45)$. This ORF started with an AUG codon at nucleotide 33 (numbered from the $5^{\prime}$ end of the vc RNA) and extended to an UAA stop codon at position 8669 . The $5^{\prime}$ noncoding region of the vc RNA was $32 \mathrm{nts}$ in length, and the $3^{\prime}$ noncoding region was $248 \mathrm{nts}$ long. The predicted $\mathrm{L}$ protein contained 2,878 amino acid (aa) with a calculated $\mathrm{M}_{\mathrm{r}}$ of $331.8 \mathrm{kDa}$.

Sequence comparisons of the terminal regions of WSMoV RNA segments. Comparisons of the $5^{\prime}$ and $3^{\prime}$ terminal regions of the $\mathrm{v}$ strand of all three RNA segments of WSMoV revealed a consensus sequence of $10 \mathrm{nts}$ (5'-AGAGCAAUCG-3') at the $5^{\prime}$ ends and a consensus sequence of 9 nts ( $5^{\prime}$-GAUUGCUCU-3') at the $3^{\prime}$ ends. The $5^{\prime}$ and $3^{\prime}$ terminal regions of each RNA segment of WSMoV contained inverted complementary sequences, similar to those found in the terminal genomic sequences of other tospoviruses. Furthermore, 18 (except the 11th position), 14 (except the 10th position), and 14 nts of the terminal sequences of the L, M, and S RNA segments were fully complementary, respectively.

The $5^{\prime}$ terminal region of the $\mathrm{v}$ L RNA of WSMoV shared a consensus sequence of $16 \mathrm{nts}$ with that of PBNV, and the $3^{\prime}$ terminal region shared a consensus sequence of $40 \mathrm{nts}$. Both termini of WSMoV v L RNA contained 9 nts identical to the terminal sequences of L RNAs of TSWV and INSV. Thus, for the v L RNAs of all known tospoviruses there was a consensus sequence of $9 \mathrm{nts}$ at both $5^{\prime}$ and $3^{\prime}$ ends. However, comparison of the $5^{\prime}$ and $3^{\prime}$ terminal sequences of the three genomic RNAs of all known tospoviruses revealed a consensus sequence of 8 nts ( $5^{\prime}$-AGAGCAAU- $3^{\prime}$ ) at their $5^{\prime}$ ends and a consensus sequence of 8 nts ( $5^{\prime}$-AUUGCUCU-3') at their $3^{\prime}$ ends. This molecular evidence supports the positioning of WSMoV in the genus Tospovirus of the family Bunyaviridae (14).

The $5^{\prime}$ noncoding sequences of L ORF on the vc L RNA of WSMoV were 32 nts in length, the same as that of PBNV and similar to those for TSWV and INSV (31 and 33 nts, respec-

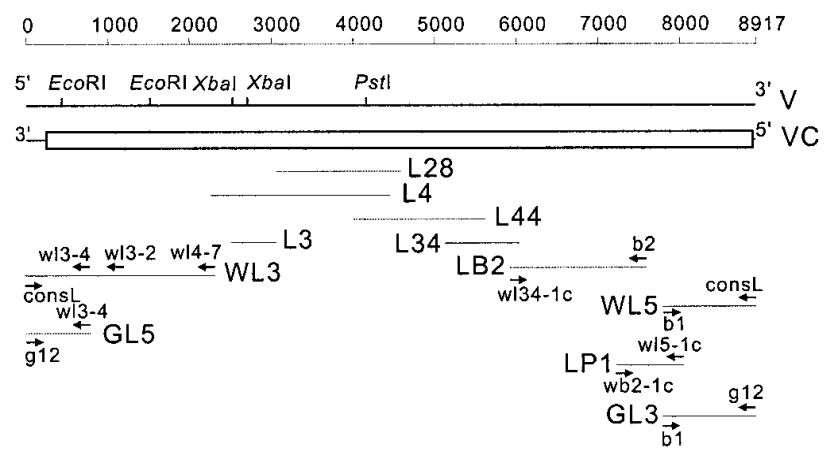

Fig. 1. Map of the DNA clones constructed from the L double-stranded RNA of Watermelon silver mottle virus by random priming and reverse transcription-polymerase chain reaction (RT-PCR). Arrows indicate the primers used for RT-PCR. The viral (v) and viral complementary (vc) strands are indicated, and the box indicates the position of the open reading frame. 
tively). In contrast, the length of the $3^{\prime}$ noncoding region of $\mathrm{L}$ ORF of WSMoV were $248 \mathrm{nts}$, similar to that of PBNV (245 nts), but different to those of TSWV and INSV (236 and 139 nts, respectively). The $5^{\prime}$ noncoding region of L ORF of WSMoV shared 59.4, 71.0, and $100 \%$ identities with those of TSWV, INSV, and PBNV, respectively, whereas the $3^{\prime}$ noncoding region of L ORF of WSMoV shared nucleotide identities of 44.9, 47.0, and $76.3 \%$ with those of TSWV, INSV, and PBNV, respectively. These results indicate that the $5^{\prime}$ noncoding regions of the vc $\mathrm{L}$ RNAs of the tospoviruses compared have similar lengths and higher degrees of sequence identity than the $3^{\prime}$ noncoding region of the vc L RNAs. The conservation of nucleotide length and greater sequence identity of the $5^{\prime}$ terminal sequences rather than the $3^{\prime}$ terminal sequences was not found when other ORFs in either M RNAs or S RNAs of the sequenced tospoviruses were compared.

Sequence identity of the $L$ protein with other tospoviruses. The L protein of WSMoV was 2,878 aa in length, with 3, 13, and 1 aa longer than those of TSWV, INSV, and PBNV, respectively. Results of comparisons of the nucleotide and amino acid sequences of each of the various ORFs encoded by the tripartite genome of WSMoV with those of TSWV, INSV, and PBNV are summarized in Table 1. The nucleotide sequence of the ORF of WSMoV coding for the L protein shared identities of 55.2, 55.6, and $81.1 \%$ with those of TSWV, INSV, and PBNV, respectively. The amino acid sequence of the WSMoV L protein shared identities of $44.3,46.5$, and $91.3 \%$ with that of TSWV, INSV, and PBNV, respectively. The amino acid sequences of the NSm, G1/G2, and NP of WSMoV shared identities of 34.4 to $83.4 \%$, 30.5 to $88.7 \%$, and 30.2 to $86.2 \%$ with those of TSWV, INSV, and
PBNV, respectively. The amino acid sequence of the NSs protein shared identities of 12.1 to $84.5 \%$ with that of TSWV, INSV, and PBNV, respectively. Thus, L protein is the most conserved protein among the three serogroups of tospoviruses compared, whereas the NSs protein is generally the most variable protein.

The alignment of the L protein of WSMoV with those of other reported tospoviruses revealed that the middle portion (aa 890 to 1559 ) of the protein was its most conserved part. This region contained the sequence motifs considered characteristic of RNAdependent RNA polymerases (30) including motif A (aa 1361 to 1377), motif B (aa 1451 to 1473 ), motif C (aa 1494 to 1504), motif D (aa 1531 to 1548 ), and motif E (aa 1549 to 1559 ). By contrast, the $\mathrm{N}$ - and $\mathrm{C}$-terminal regions of the protein were variable, and the very acidic carboxyl terminus of the TSWV L protein (5 glutamate and 5 aspartate residues out of 15) was not present in the L proteins of WSMoV, PBNV, and INSV.

Phylogenetic relationships of the $L$ proteins in the family Bunyaviridae. The tospovirus $\mathrm{L}$ proteins were further aligned against those reported for other viruses in the family Bunyaviridae. Several conserved amino acid regions were found throughout the middle portion of the $\mathrm{L}$ proteins, including the five characteristic RNA-dependent RNA polymerase core motifs, as described by Poch et al. (30) and Muller et al. (27), i.e., DXXKW (motif A), QGXXXXXSS (motif B), SDD (motif C), K (motif D), and EXXS (motif E) (Fig. 2).

The results of phylogenetic relationships of the $\mathrm{L}$ proteins of WSMoV and the reported viruses in the family Bunyaviridae revealed that WSMoV, PBNV, TSWV, and INSV of the genus Tospovirus are clustered in a separate group in the family Bunyavirdae (data not shown). Our results support that the genus Tospo-

TABLE 1. Comparison of individual genes and encoded proteins of Watermelon silver mottle virus (WSMoV) with those of Peanut bud necrosis virus (PBNV), Tomato spotted wilt virus (TSWV), and Impatiens necrotic spot virus (INSV) ${ }^{\mathrm{a}}$

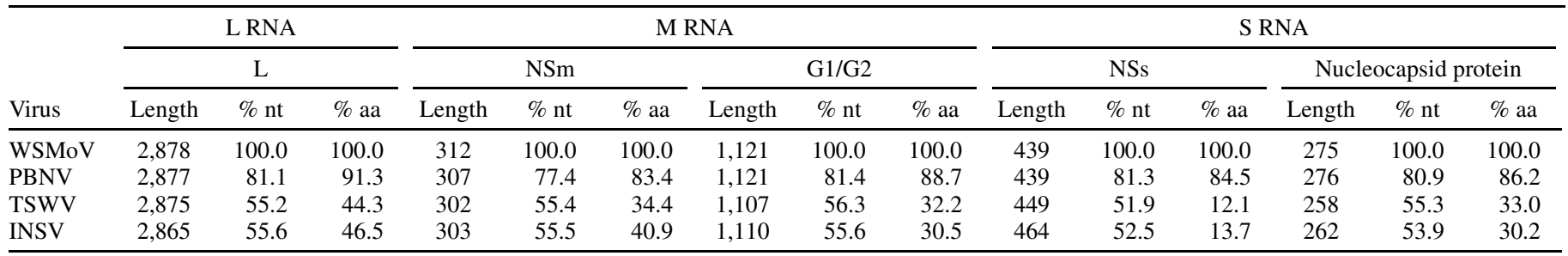

${ }^{a}$ Length $=$ the number of amino acids. $\% \mathrm{nt}=$ nucleotide identity of the open reading frame to that of WSMoV. $\%$ aa $=$ amino acid identity of the encoded protein to that of WSMoV. L RNA encodes putative RNA polymerase L protein on the viral complementary strand. Ambisense M RNA encodes nonstructural protein $\mathrm{M}(\mathrm{NSm})$ and glycoproteins precursor G1/G2 protein, and ambisense S RNA encodes nonstructural protein S (NSs) and nucleocapsid protein. The sources and GenBank codes of the nucleotide and the amino acid sequences: WSMoV, M (X78556) (7) and S (U78734) (50); PBNV, L (AF025538) (16), M (U42555) (35), and S (U27809) (34); TSWV, L (D10066) (9), M (S48091) (17), and S (AF135036) (10); INSV, L (X93218) (45), M (M74904) (23), and S (D00914) (8).

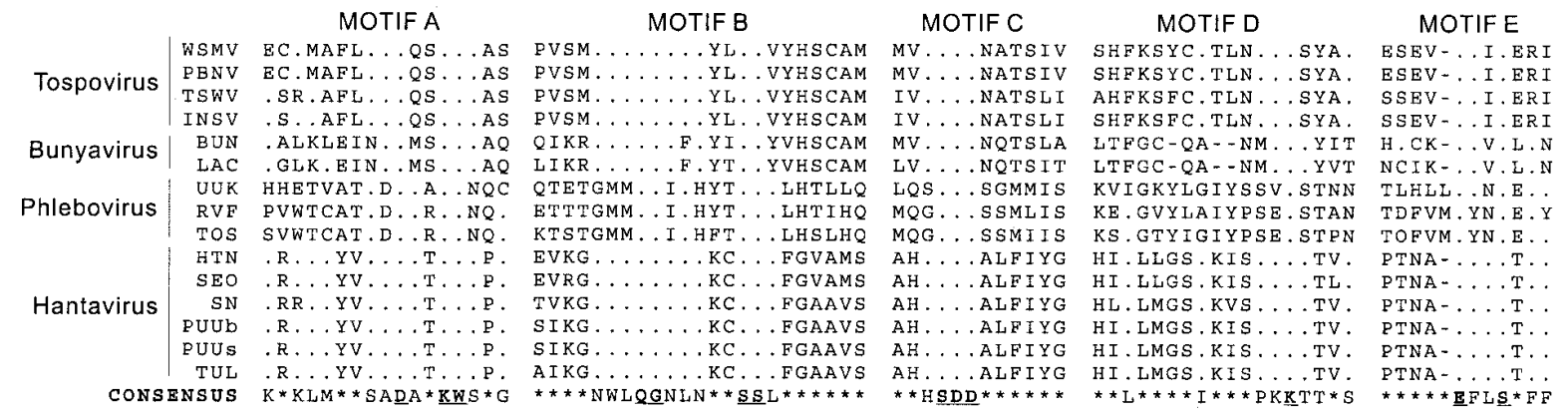

Fig. 2. Sequences of the conserved regions of the L proteins of the viruses in the family Bunyaviridae identified as polymerase motifs. Conserved amino acids were identified using the PILEUP options of the Genetics Computer Group package (Genetics Computer Group, Madison, WI). Dots indicate amino acids that are conserved in at least 8 of the 15 species and encompass at least two genera of the family Bunyaviridae. Stars indicate the different residues, and hyphens indicate gaps. Underlined amino acids denote fully conserved residues within each motif. WSMoV = Watermelon silver mottle virus; sequences were obtained from the EMBL Database with the following accession codes: PBNV = Peanut bud necrosis virus, AF025538 (16); TSWV = Tomato spotted wilt virus, D10066 (9); INSV = Impatiens necrotic spot virus, X93218 (45); BUN = Bunyamwera, X14384 (11); LAC = La Crosse, U12396 (32); UUK = Unkuniemi, D10759 (13); RVF = Rift Valley fever, X56464 (27); TOS = Toscana, X68414 (1); HTN = Hantaan, X55901 (36); SEO = Seoul, X56492 (4); SN = Sin Nombre, L37901 (6); PUUb = Puumala strain Bashkiria, M63194 (38); PUUs = Puumala strain Sotkamo, Z66548 (29); and TUL = Tula, AJ005637 (21). 
virus has a closer relationship with the genus Bunyavirus than with the genera Phlebovirus and Hantavirus, as previously reported $(6,32,45)$.

Detection of tospoviruses from five different serogroups by RT-PCR with degenerate primers. Two pairs of degenerate primers were designed from the conserved regions of the $\mathrm{L}$ genes of WSMoV and those of other reported tospoviruses. The sequences and the positions of the target regions of WSMoV L RNA are shown in Figure 3. The DNA fragments obtained by RT-PCR are shown in Figure 4A and B, respectively. When the primer pair gL3637/gL4435c was used, a 0.81-kbp DNA fragment was amplified from the total RNAs extracted from plants infected with individual tospoviruses, except PCFV, from which only a $0.38-\mathrm{kbp}$ fragment was obtained (Fig. 4A). When the primer pair gL3637/ gL4510c was used, a 0.89-kbp DNA fragment was amplified from total RNAs extracted from plants infected with individual tospoviruses, except PCFV, from which a fragment of only $0.46 \mathrm{kbp}$ was generated (Fig. 4B). No products were obtained when total RNAs from healthy plants or plants infected with viruses from other genera, namely ORSV, PVX, PRSV, SLRV, CMV, RGSV, and RSV, were tested (Fig. 4A and B). The length of the 0.81- and $0.89-\mathrm{kbp}$ fragments matched well with the expected regions flanked by the primers, and it was suggested that they were specific amplicons derived from L RNAs of the tospoviruses.

Confirmation by restriction enzyme digestion. The amplified fragments of TSWV, GRSV, INSV, WSMoV, HT-1, and PCFV were hydrolyzed with HindIII, XbaI, and EcoRI, and the patterns of the fragments obtained are shown in Figure 5A and B. The unique patterns indicated that no cross contamination had occurred. Moreover, the patterns of TSWV (9), INSV (45), and WSMoV were identical to the predicted patterns derived from the known sequences of the amplified regions. The patterns of WSMoV and HT-1 were similar in XbaI and EcoRI digestions, but differed in the HindIII digestion. The patterns obtained from the PCFV fragments were distinct from those of the other tospoviruses.

Verification by sequencing. Individual fragments amplified by the primer pair gL3637/gL4510c were cloned and sequenced to analyze their primary structures. Alignment of the amino acid sequences of the five 0.89-kbp amplicons of TSWV, GRSV, INSV, WSMoV, and HT-1 revealed that they had the sequences of the targeted conserved regions of the $\mathrm{L}$ gene, including the conserved motif A, B, and C (Fig. 6). The sequences of the amplicons from TSWV (9), INSV (45), and WSMoV were the same as the

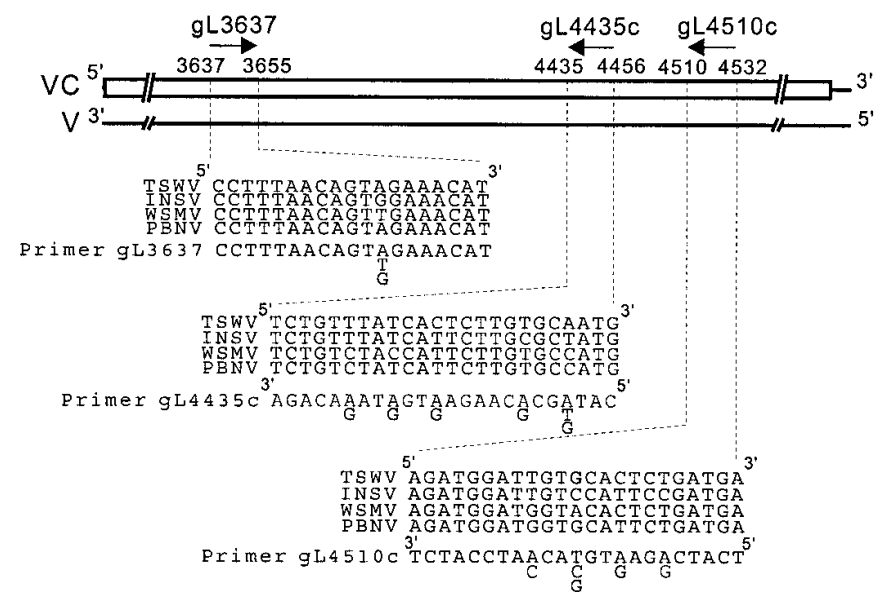

Fig. 3. Sequences of degenerate primers designed from the sequences of the conserved regions of L RNAs of Tomato spotted wilt virus (TSWV) (9), Impatiens necrotic spot virus (INSV) (45), Watermelon silver mottle virus (WSMoV), and Peanut bud necrosis virus (PBNV) (16). Arrows indicate regions where degenerate primers bind. Numbers indicate the nucleotide positions targeted and correspond to the viral complementary (vc) strand of WSMoV L RNA. reported sequences of the corresponding regions of the $\mathrm{L}$ proteins. The sequences of GRSV and HT-1 were not identical to the reported ones, but aligned well with the known ones. The results verified that the RT-PCR products came from the span targeted by the degenerate primer pairs used. Although the 0.46- and the 0.38-kbp fragments of PCFV were smaller than those of the others, they also covered the specific domains $\mathrm{A}, \mathrm{B}$, and $\mathrm{C}$ of the $\mathrm{L}$ protein, but the region corresponding to the $\mathrm{N}$-terminal half of the amplicons from the other five tospoviruses was missing (Fig. 6).

Detection of tospoviruses in field samples. When the primer pairs gL3637/gL4435c and gL3637/gL4510c were used for detection of the presence of tospoviruses in naturally infected plants, 0.8- and 0.9-kbp DNA fragments (Fig. 7A), respectively, were amplified from the total RNAs extracted from 12 diseased samples of watermelon, 8 samples of wax gourd, and 3 samples of lisianthus. No products were obtained from total RNAs of healthy controls. In addition, when the WSMoV N-gene-specific primers were used for detection, a DNA fragment of $0.7 \mathrm{kbp}$ was amplified from all diseased samples of watermelon and wax gourd that were positively detected by the two pairs of degenerate primers, but no product was obtained when TSWV N-gene-specific primers were used (Fig. 7B). An amplified fragment of $1.0 \mathrm{kbp}$ was noticed from the three diseased samples of lisianthus when they were detected by the TSWV N-gene-specific primers, but no product was found when WSMoV N-gene-specific primers were used (Fig. 7B). Among the samples that were positive to WSMoV N-gene-specific primers, only 10 samples of watermelon and 7 samples of wax gourd were ELISA positive when tested against WSMoV NP antiserum, but all of them were negative to TSWV NP antiserum (Fig. 7C). These results indicated that detection by RT-PCR for WSMoV in field samples is more sensitive than indirect ELISA. However, the three samples
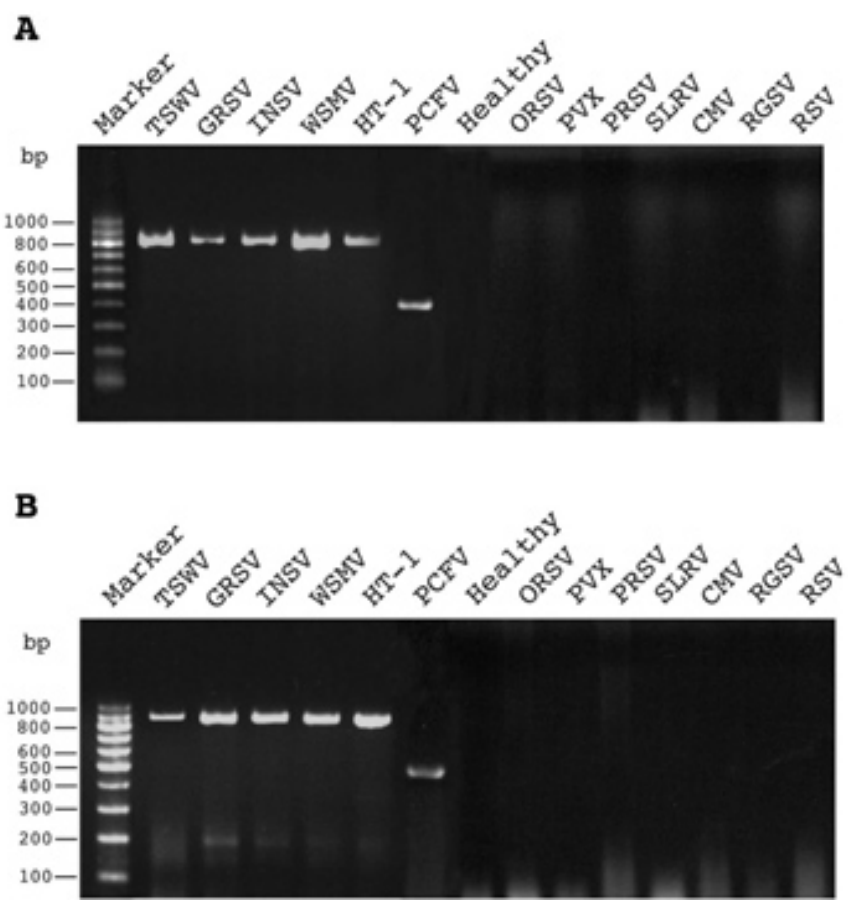

Fig. 4. Reverse transcription-polymerase chain reaction (RT-PCR) analyses of tospoviruses from different serogroups and plant viruses of other genera, using primer pairs to the conserved regions of the L genes A, gL3637/ gL4435c and B, gL3637/gL4510c. RT-PCR was performed using total RNAs extracted from plants infected with individual viruses as templates. TSWV = Tomato spotted wilt virus; GRSV = Groundnut ringspot virus; INSV = Impatiens necrotic spot virus; WSMV = Watermelon silver mottle virus; HT-1 = high temperature-recovered tospovirus; PCFV = Peanut chlorotic fan-spot virus; ORSV = Odontoglossum ringspot virus; $\mathrm{PVX}=$ Potato virus $X$; $\mathrm{PRSV}=$ Papaya ringspot virus $; \mathrm{CMV}=$ Cucumber mosaic virus; $\mathrm{RGSV}=$ Rice grassy stunt virus; and RSV = Rice stripe virus. 
that were positive to TSWV N-gene-specific primers only reacted positively with TSWV NP antiserum and not with WSMoV NP antiserum (Fig. 7C). Our results of RT-PCR detections and indirect ELISA revealed that the 12 watermelon and 8 wax gourd
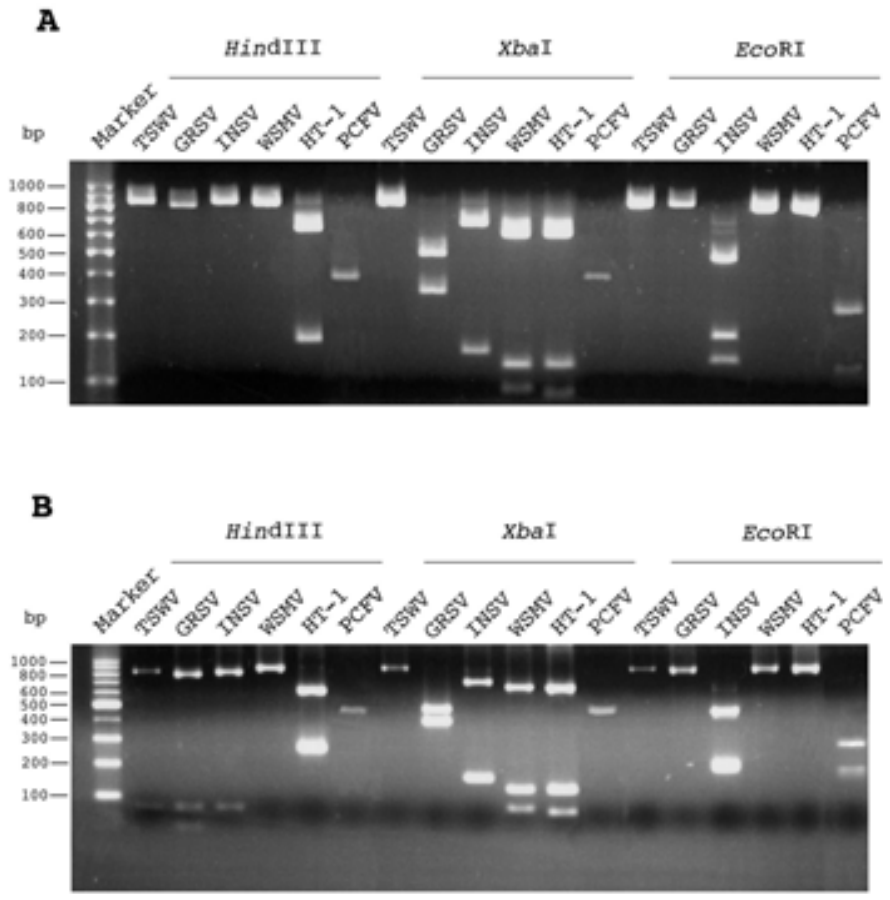

Fig. 5. Restriction enzyme analyses of reverse transcription-polymerase chain reaction products amplified from tospoviruses in different serogroups using degenerate primers pairs to the conserved regions of $\mathrm{L}$ genes, $\mathbf{A}$, gL3637/gL4435c and B, gL3637/gL4510c. The restriction enzymes HindIII, $X b a \mathrm{I}$, and EcoRI were used. Combinations of the viruses and enzymes are indicated above each set. TSWV = Tomato spotted wilt virus; GRSV = Groundnut ringspot virus; INSV = Impatiens necrotic spot virus; WSMoV = Watermelon silver mottle virus; HT-1 = high temperature-recovered tospovirus; and PCFV = Peanut chlorotic fan-spot virus. samples were infected by WSMoV, and the 3 lisianthus samples were infected by TSWV.

\section{DISCUSSION}

The L RNA of WSMoV was 8,917 nts in length. It is similar to those of other reported Tospovirus species (8,776 to 8,911 nts), but significantly longer than the L RNAs of viruses of the genera Bunyavirus (6,875 to 6,980 nts), Phlebovirus (6,404 to 6,606 nts), and Hantavirus (6,530 to 6,562 nts) of the family Bunyaviridae, except that of Dugbe virus $(18,855 \mathrm{nts})$ of the genus Nairovirus (25), which has an L protein (4,036 aa) too long to be analyzed by the programs we used. However, the L protein of this nairovirus also contains five polymerase motifs common to members of the family Bunyaviridae (25).

The $5^{\prime}$ and $3^{\prime}$ ends of WSMoV L RNA are complementary and can be folded into a panhandle structure like those of the $\mathrm{M}$ and $\mathrm{S}$ RNA, a typical feature of all segmented negative-stranded viruses. This structure is thought to be required in the process of initiation and regulation of viral transcription, replication, and encapsidation (46). The nucleotide lengths of the compared $5^{\prime}$ noncoding regions of the vc strands of L ORFs of tospoviruses that are complementary to the $3^{\prime}$ terminal sequences of the $\mathrm{v}$ strands, differed only in 1 to 2 nts, in contrast to the variable lengths of the noncoding regions of other ORFs. We suggest that although the $5^{\prime}$ and $3^{\prime}$ terminal regions of M and S RNA are essential for polymerase binding, the regulatory sequences for the expression of the different ORFs of $\mathrm{M}$ and $\mathrm{S}$ RNAs make the terminal sequences variable because of their ambisense characteristic. The L RNA is of negative polarity, and the $3^{\prime}$ terminal sequence of the $\mathrm{v}$ strand is essential for recognition by the RNA polymerase to generate the message to express L protein for initiation of replication. Given the remarkably conserved nature of the putative functional motifs of tospovirus L proteins, it is reasonable that the $3^{\prime}$ terminal sequence of the $\mathrm{v}$ strand also is conserved for the recognition or binding of the L protein to synthesize positive sense L RNA.

Because the $\mathrm{L}$ proteins of the four known members of tospoviruses have some very conserved regions, it would be expected that degenerate primers could be designed to detect tospoviruses from
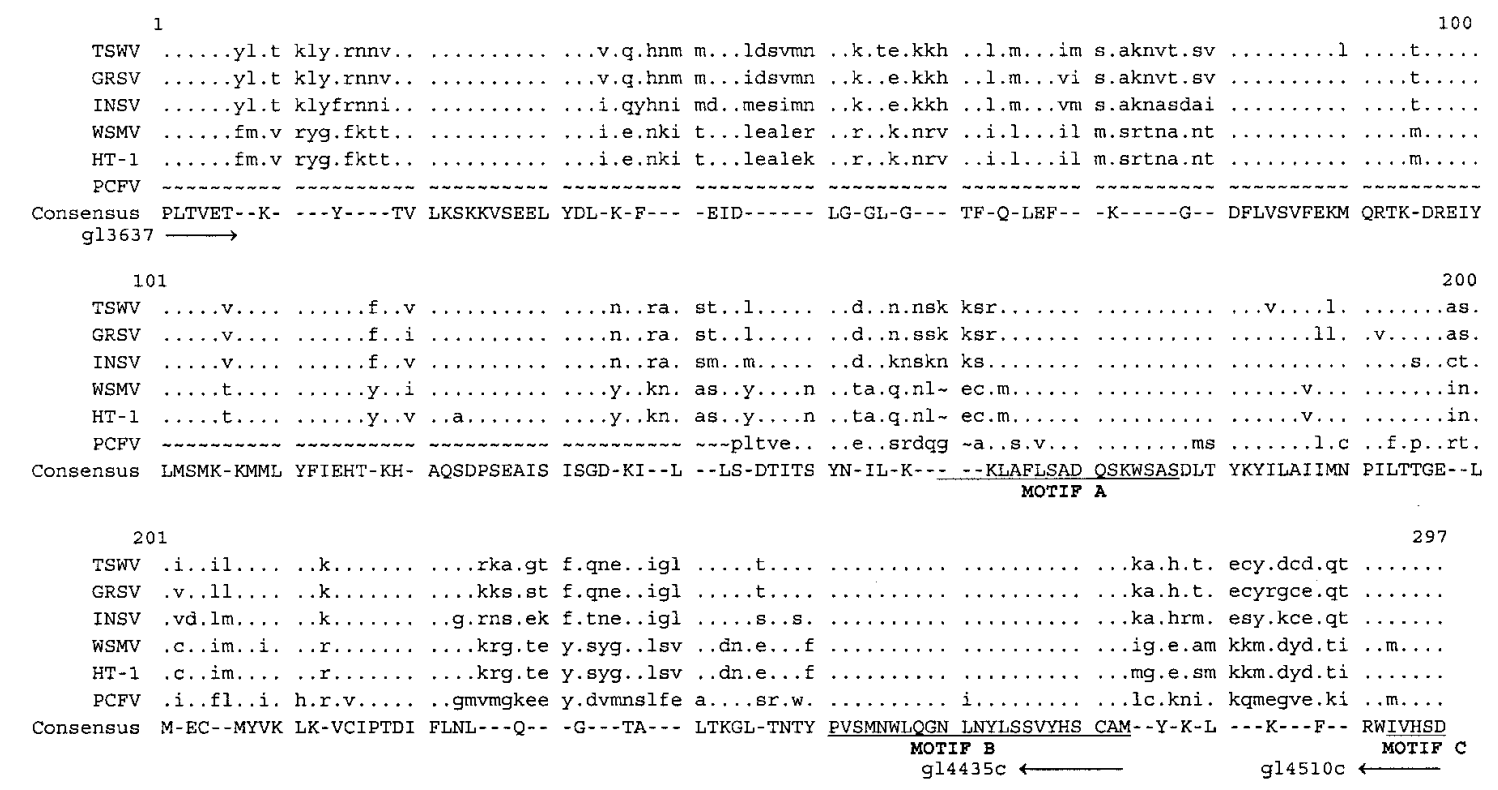

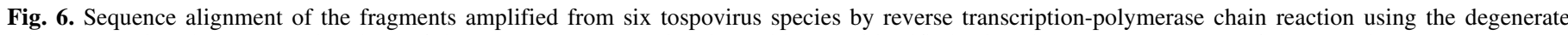

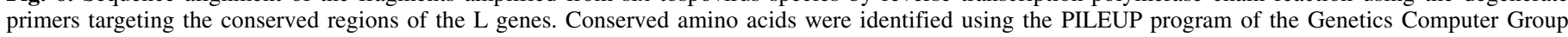

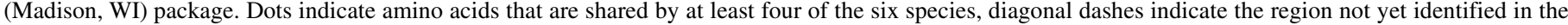

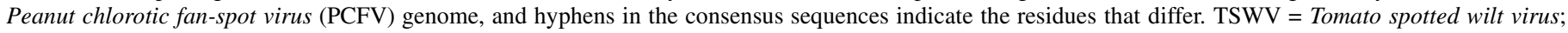

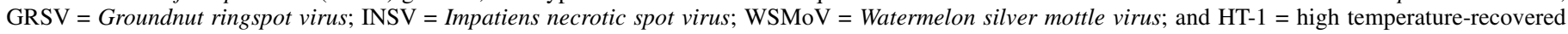
tospovirus. 
different serogroups. In this paper, two pairs of degenerate primers targeting the conserved regions of the L genes were successfully utilized to amplify two corresponding regions of the L genes of tospoviruses from the four common tospovirus serogroups. Although PCFV, which is distinct from the four serogroups (48), yielded fragments smaller than those of the others, the amplicons also covered specific domains $\mathrm{A}, \mathrm{B}$, and $\mathrm{C}$ of the $\mathrm{L}$ protein, but lacked the $\mathrm{N}$-terminal region of domain $\mathrm{A}$. Because the virus population of PCFV used for inoculation was derived from single lesion passages and the presence of the virus was doubly checked by ELISA, we believe that the amplicon represents the conserved part of the L gene of PCFV. Whether the missing region is absent in the L RNA of PCFV or the gl3637 primer mismatched remains to be further investigated. Moreover, the possibility that the shorter amplicons resulted from defective L RNA cannot be excluded.

Six viruses from the genera Tobamovirus, Potexvirus, Potyvirus, Nepovirus, Cucumovirus, and Tenuivirus were used to test the

A
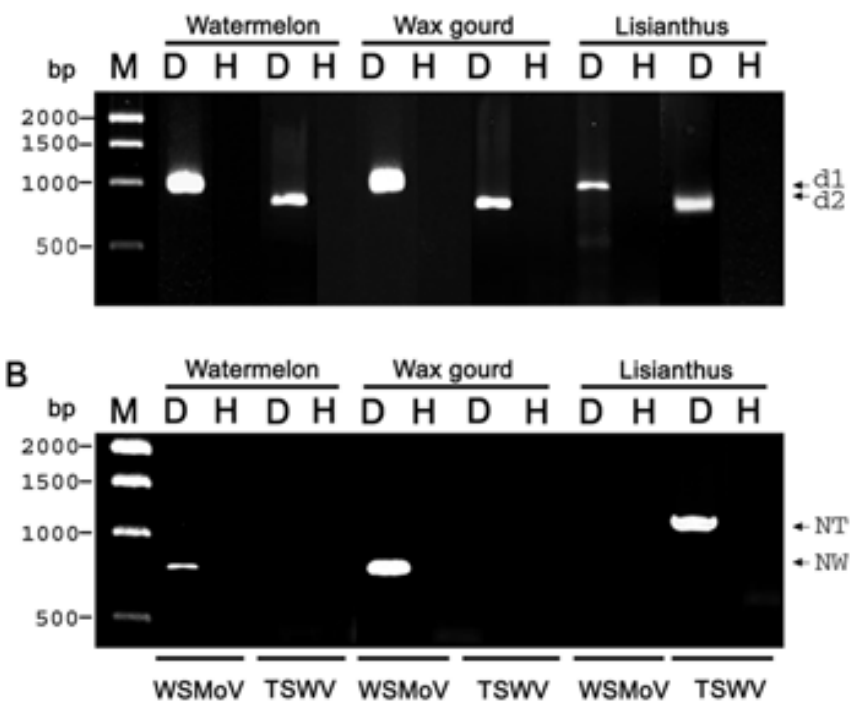

C

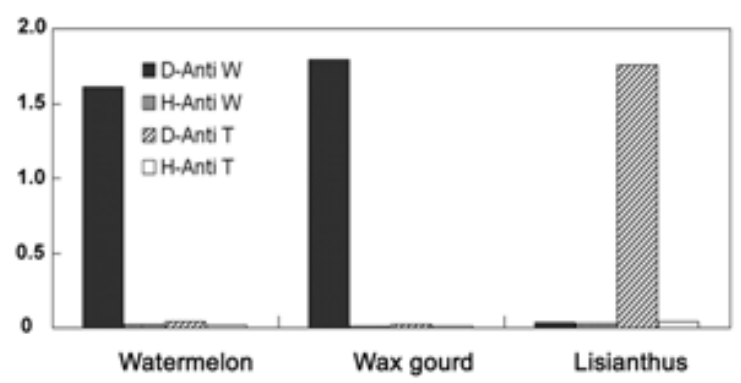

Fig. 7. Detection of tospoviruses of distinct serogroups in field samples by reverse transcription-polymerase chain reaction (RT-PCR) using the degenerate primer pairs designed from the conserved region of the L RNAs of tospoviruses. The total RNAs extracted from field samples and healthy plants of watermelon, wax gourd, and lisianthus were analyzed by RT-PCR using degenerate primer pairs gL3637/gL4510c and gL3637/gL4435c, derived from $\mathbf{A}, \mathrm{L}$ genes of the tospoviruses and $\mathbf{B}, \mathrm{N}$-gene primers specific to Watermelon silver mottle virus (WSMoV) or Tomato spotted wilt virus (TSWV). C, The crude extracts from field samples and healthy plants were also analyzed by indirect enzyme-linked immunosorbent assay (ELISA) using the antiserum to the nucleocapsid protein (NP) of WSMoV or TSWV. The host plants used are indicated in order from left to right. M, D, and $\mathrm{H}$ represent DNA markers, diseased sample, and healthy control, respectively. A, $\mathrm{d} 1$ and $\mathrm{d} 2$ represent the products amplified by degenerate primer pairs gL3637/gL4510c and gL3637/gL4435c, respectively. B, NT and NW represent the products amplified by TSWV N-gene and WSMoV N-genespecific primers, respectively. C, Anti W and Anti T represent the antisera to the NPs of WSMV and TSWV, respectively. specificity of the primer sets. Among plant virus genera, the genus Tenuivirus, which has a negative sense and ambisense multipartite genome, is considered closer to the genus Tospovirus. Previous reports indicated that the amino acid in major RNA-dependent RNA polymerase modules (motifs A to E) of tenuviruses RGSV (41) and RSV (42) are more conserved with those of phlebovirus than those of other genera in the family Bunyaviridae. Comparison of putative RNA polymerase of WSMoV with those of RGSV and RSV showed that they only share 16.7 and $29.1 \%$ amino acid identities, respectively. When the amino acid in the motifs of the L proteins of tospoviruses TSWV, INSV, WSMoV, and PBNV were compared with those of tenuiviruses RGSV and $\mathrm{RSV}$, it was found that there are only limited residues conserved, such as the amino acid resides SXDXSKW in motif A, DGXLXYXSSXXH in motif B, SDD in motif C, KS in motif D, and EFXXE in motif $\mathrm{E}$ (data not shown). The low nucleotide identities of the degenerate primers with the corresponding regions of tenuiviruses explain why total RNAs from plants infected with RGSV or RSV were not amplified in RT-PCR tests using the primer sets. These results indicated that the primers appear specific to tospoviruses. Whether these two pairs of degenerate primers can detect viruses in the other genera in the family Bunyaviridae needs to be investigated. Nevertheless, because the other genera of the family Bunyaviridae infect only animals, it would not create ambiguity when the primer sets are used for detecting plant tospoviruses (15).

The digestion patterns by restriction enzyme and the sequence analysis indicated that the amplified fragments were identical to the targeted regions in the individual $\mathrm{L}$ genes. The patterns may be used to differentiate tospoviruses in different serogroups. In this investigation, we have shown that the degenerate primer pairs designed from the conserved region of L protein, not only, can be used for detection of tospoviruses in five different serogroups, but also able to detect field samples infected with WSMoV or TSWV. Because GRSV and INSV have not been recorded in Taiwan and PCFV is only occasionally noticed, field samples with these three tospoviruses were not available for RT-PCR assays. Whether the degenerate primers can be used for detecting tospoviruses in all other Tospovirus spp. remains to be tested. Because of broad host ranges, complicated symptomatology, and serological diversity, it is very helpful to develop reliable tools for detection of tospoviruses. We suggest that the use of the tospovirus-specific degenerate primers developed in this study, combined with the specific probes to $\mathrm{N}$ genes or specific antisera to NPs, should provide a fast and accurate way for identifying and diagnosing tospoviruses. For further application, monoclonal antibodies against the highly conserved motifs of L proteins are currently being prepared in our laboratory and may be used as an alternative broad-spectrum serological tool for detecting tospoviruses in different serogroups.

\section{ACKNOWLEDGMENTS}

This study was partly supported by a cooperative grant sponsored by the U.S. Department of Agriculture and the Council of Agriculture of the Republic of China in Taiwan (88AST-1.4-FAD-0.1).

\section{LITERATURE CITED}

1. Accardi, L., Gro, M. C., Di Bonito, P., and Giorgi, C. 1993. Toscana virus genomic L segment: Molecular cloning, coding strategy and amino acid sequence in comparison with other negative strand RNA viruses. Virus Res. 27:119-131.

2. Adam, G., Yeh, S. D., Reddy, D. V. R., and Green, S. K. 1993. Serological comparison of tospovirus isolates from Taiwan and India with impatiens necrotic spot virus and serogroups of tomato spotted wilt virus. Arch. Virol. 130:237-250.

3. Adkins, S., Quadt, R., Choi, T. J., Ahlquist, P., and German, T. 1995. An RNA-dependent RNA polymerase activity associated with virions of tomato spotted wilt virus, a plant- and insect-infecting bunyavirus. Virology 207:308-311. 
4. Antic, D., Lim, B. U., and Kang, C. Y. 1991. Nucleotide sequence and coding capacity of the large (L) genomic RNA segment of Seoul 80-39 virus, a member of the hantavirus genus. Virus Res. 19:59-66.

5. Bailey, J. M., and Davidson, N. 1976. Methylmercury as a reversible denaturing agent for agarose gel electrophorsis. Anal. Biochem. 70:75-85.

6. Chizhikov, V. E., Spiropoulou, C. F., Morzunov, S. P., Montoe, M. C., Peters, C. J., and Nichol, S. T. 1995. Complete genetic characterization and analysis of isolation of Sin Nombre virus. J. Virol. 69:8132-8136.

7. Chu, F.-H., and Yeh, S.-D. 1998. Comparison of ambisense M RNA of watermelon silver mottle virus with other tospoviruses. Phytopathology 88:351-358.

8. de Haan, P., de Avila, A. C., Kormelink, R., Westerbroek, A., Gielen, J. J. L., Peters, D., and Goldbach, R. W. 1992. The nucleotide sequence of the S RNA of impatiens necrotic spot virus, a novel tospovirus. FEBS Lett. 306:27-32.

9. de Haan, P., Kormelink, R., de Oliveira Resende, R., Van Poelwijk, F., Peters, D., and Goldbach, R. 1991. Tomato spotted wilt virus L RNA encodes a putative RNA polymerase. J. Gen. Virol. 71:2207-2216.

10. de Haan, P., Wagemarker, L., Peters, D., and Goldbach, R. 1990. The S RNA segment of tomato spotted wilt virus has an ambisense character. J. Gen. Virol. 71:1001-1007.

11. Elliott, R. M. 1989. Nucleotide sequence analysis of the large (L) genomic segment of Bunyamwera virus, the prototype of the family Bunyaviridae. Virology 173:426-436.

12. Elliott, R. M. 1990. Molecular biology of the Bunyaviridae. J. Gen. Virol. 71:501-522.

13. Elliott, R. M., Dunn, E., Simons, J. F., and Pettersson, R. F. 1992. Nucleotide sequence and coding strategy of Uukuniemi virus L RNA segment. J. Gen. Virol. 73:1745-1752.

14. Francki, R. I. B., Fauquet, C. M., Knudson, D. L., and Brown, F. 1991. Classification and nomenclature of viruses. Fifth report of the International Committee on Taxonomy of Viruses. Arch. Virol. Suppl. 2:450.

15. German, T. L., Ullman, D. E., and Moyer, J. W. 1992. Tospoviruses: Diagnosis, molecular biology, phylogeny, and vector relationships. Annu. Rev. Phytopathol. 30:315-348.

16. Gowda, S., Satyanarayana, T., Naidu, R. A., Mushegian, A., Dawson, W. O., and Reddy, D. V. R. 1998. Characterization of the large (L) RNA of peanut bud necrosis tospovirus. Arch. Virol. 143:2381-2390.

17. Kormelink, R., de Haan, P., Meurs, C., Peters, D., and Goldbach, R. 1992. The nucleotide sequence of the M RNA segment of tomato spotted wilt virus, a bunyavirus with two ambisense RNA segments. J. Gen. Virol. 73:2795-2804.

18. Kormelink, R., de Haan, P., Peters, D., and Goldbach, R. 1992. Viral RNA synthesis in tomato spotted wilt virus-infected Nicotiana rustica plants. J. Gen. Virol. 73:687-693.

19. Kormelink, R., Kitajima, E. W., de Haan, P., Zuidema, D., Peters, D., and Goldbach, R. 1991. The nonstructural protein (NSs) encoded by the ambisense S RNA segment of tomato spotted wilt virus is associated with fibrous structures in infected plant cells. Virology 181:459-468.

20. Kormelink, R., Storms, M., Van Lent, J., Peters, D., and Goldbach, R. 1994. Expression and subcellular location of the NSm protein of tomato spotted wilt virus (TSWV), a putative viral movement protein. Virology 200:56-65.

21. Kukkonen, S. K. J., Vaheri, A., and Plyusis, A. 1998. Completion of the Tula hantavirus genome sequence: properties of the $\mathrm{L}$ segment and heterogeneity found in the $3^{\prime}$ termini of S and L genome RNAs. J. Gen. Virol. 79:2615-2622.

22. Law, M. D., Speck, J., and Moyer, J. W. 1991. Nucleotide sequence of the $3^{\prime}$ non-coding region and $\mathrm{N}$ gene of the $\mathrm{S}$ RNA of a serologically distinct tospovirus. J. Gen. Virol. 72:2597-2601.

23. Law, M. D., Speck, J., and Moyer, J. W. 1992. The M RNA of impatiens necrotic Tospovirus (Bunyaviridae) has an ambisense genomic organization. Virology 188:732-741.

24. Maniatis, T., Fritsch, E. F., and Sambrook, J. 1982. Molecular Cloning: A Laboratory Manual. Cold Spring Harbor Laboratory, Cold Spring Harbor, NY.

25. Marriott, A. C., and Nuttall, P. A. 1996. Large RNA segment of Dugbe nairovirus encodes the putative RNA polymerase. J. Gen. Virol. 77:1775-1780.

26. Mohamed, N. A., Randles, J. W., and Francki, R. I. B. 1973. Protein composition of tomato spotted wilt virus. Virology 56:12-21.

27. Muller, R., Poch, O., Delarue, M., Bishop, D. H. L., and Bouloy, M. 1994. Rift valley fever virus L segment: Correction of the sequence and possible functional role of newly identified regions conserved in RNAdependent polymerases. J. Gen. Virol. 75:1345-1352.

28. Murphy, F. A., Fauquet, C. M., Bishop, D. H. L., Ghabrial, S. A., Jarvis, A. W., Martelli, G. P., Mayo, M. A., and Summers, M. D. (ed.) 1995.
Virus Taxonomy. Pages 300-315 in: Classification and Nomenclature of Viruses. Sixth Report of the International Committee on Taxonomy of Viruses. Springer-Verlag, New York.

29. Piiparinen, H., Vapalahti, O., Plyusnin, A., Vaheri, A., and Lankinen, H. 1997. Sequence analysis of the Puumala hantavirus Sotkamo strain L segment. Virus Res. 51:1-7.

30. Poch, O., Sauvaget, I., Delarue, M., and Tordo, N. 1989. Identification of four conserved motifs among the RNA-dependent polymerases encoding elements. Eur. Mol. Biol. Organ. J. 8:3867-3874.

31. Reddy, D. V. R., Ratna, A. S., Sudarshana, M. R., Poul, F., and Kumar, I. K. 1992. Serological relationships and purification of bud necrosis virus, a tospovirus occurring in peanut (Arachis hypogaea L.) in India. Ann. Appl. Biol. 120:279-286.

32. Roberts, A., Rossier, C., Kolakofsky, D., Nathanson, N., and GonzalezScarano, F. 1995. Completion of the La Crosse virus genome sequence and genomic comparisons of $\mathrm{L}$ proteins of the Bunyaviridae. Virology 206:742-745.

33. Sanger, F., Nicklen, S., and Coulson, A. R. 1977. DNA sequencing with chain-terminating inhibitors. Proc. Natl. Acad. Sci. USA 83:571-579.

34. Satyanarayana, T., Mitchell, S. E., Reddy, D. V. R., Brown, S., Kresovich, S., Jarret, R., Naidu, R. A., and Demski, J. W. 1996. Peanut bud necrosis tospovirus S RNA: Complete nucleotide sequence, genome organization and homology to other tospoviruses. Arch. Virol. 141:8598.

35. Satyanarayana, T., Mitchell, S. E., Reddy, D. V. R., Kresovich, S., Jarret, R., Naidu, R. A., Gowda, S., and Demski, J. W. 1996. The complete nucleotide sequence and genome organization of the M RNA segment of peanut bud necrosis tospovirus and comparison with other tospoviruses. J. Gen. Virol. 77:2347-2352.

36. Schmaljohn, C. S. 1990. Nucleotide sequence of the L genome segment of Hantaan virus. Nucleic Acids Res. 18:6728.

37. Singh, S. J., and Krishnareddy, M. 1996. Watermelon bud necrosis: A new tospovirus disease. Acta Hortic. 431:68-77.

38. Stohwasser, R., Raab, K., Darai, G., and Bautz, E. K. F. 1991. Primary structure of the large (L) RNA segment of nephropathia epidemica vieus atrain Hallnas B1 coding for the viral RNA polymerase. Virology 183:386-391.

39. Storms, M. M. H., Kormelink, R., Peter, D., Van Lent, J. W. M., and Goldbach, R. W. 1995. The nonstructural NSm protein of tomato spotted wilt virus induces tubular structures in plant and insect cells. Virology 214:485-493.

40. Tas, P. W. L., Boerjan, M. L., and Peters, D. 1977. The structural proteins of tomato spotted virus. J. Gen. Virol. 36:267-279.

41. Toriyama, S., Kimishima, T., Takahashi, M., Shimizu, T., Minaka, N., and Akutsu, K. 1998. The complete nucleotide sequence of the rice grassy stunt virus genome and genomic comparisons with viruses of the genus Tenuivirus. J. Gen. Virol. 79:2051-2058.

42. Toriyama, S., Takahashi, M., Sano, Y., Shimizu, T., and Ishihama, A. 1994. Nucleotide sequence of RNA1, the largest segment of rice stripe virus, the prototype of the tenuiviruses. J. Gen. Virol. 75:3569-3579.

43. Ullman, D. E., German, T. L., Sherwood, J. L., Westcot, D. M., and Cantone, F. A. 1993. Tospovirus replication in insect vector cells: Immunocytochemical evidence that the nonstructural protein encoded by the $\mathrm{S}$ RNA of tomato spotted wilt tospovirus is present in thrips vector cells. Phytopathology 83:456-463.

44. van Den Hurk, J., Tas, P. W. L., and Peters, D. 1977. The ribonucleic acid of tomato spotted wilt virus. J. Gen. Virol. 36:81-91.

45. van Poelwijk, F., Prins, M., and Goldbach, R. 1997. Completion of the impatiens necrotic spot virus genome sequence and genetic comparison of the $\mathrm{L}$ proteins within the family Bunyaviridae. J. Gen. Virol. 78: 543-546.

46. Wertz, G. W., Whelan, S., LeGrone, A., and Ball, L. A. 1994. Extent of terminal complementarity modulates the balance between transcription and replication of vesicular stomatitis virus RNA. Proc. Natl. Acad. Sci. USA 91:8587-8591.

47. Yeh, S.-D., and Chang, T.-F. 1995. Nucleotide sequence of the $\mathrm{N}$ gene of watermelon silver mottle virus, a proposed new member of the genus Tospovirus. Phytopathology 85:58-64.

48. Yeh, S. D., Chao, C. H., Cheng, Y. H., and Chen, C. C. 1996. Serological comparison of four distinct tospoviruses by polyclonal antibodies to purified nucleocapsid proteins. Acta Hortic. 431:122-134.

49. Yeh, S.-D., Lin, Y.-C., Cheng, Y.-H., Jih, C.-L., Chen, M.-J., and Chen, C.-C. 1992. Identification of tomato spotted wilt-like virus infecting watermelon in Taiwan. Plant Dis. 76:835-840.

50. Yeh, S. D., Sun, I. J., Ho, H. M., and Chang, T. F. 1996. Molecular cloning and nucleotide sequence analysis of the S RNA of watermelon silver mottle tospovirus. Acta Hortic. 431:244-260. 